\title{
Collembola Poduromorpha de áreas preservadas e impactadas do litoral sudeste do Brasil
}

\author{
Liliane H. Fernandes \& Maria C. de Mendonça
}

Departamento de Entomologia, Museu Nacional. Quinta da Boa Vista, São Cristóvão, 20940-040 Rio de Janeiro, Rio de Janeiro, Brasil. E-mail: liliane@acd.ufrj.br; mcleide@acd.ufrj.br

\begin{abstract}
Collembola Poduromorpha from preserved and polluted areas in the Brazilian southeast littoral. The present article increases the knowledge of Poduromorpha species to 34 in the Brazilian littoral thought the record of Paraxenylla piloua Thibaud \& Weiner, 1997; Xenylla welchi Folsom, 1916; Friesea claviseta (Axelson, 1900); Friesea magnicornis Denis, 1931 and Friesea mirabilis (Tullberg, 1871) in Maricá, Rio de Janeiro State. The morphological study of the 23 species allowed attach new characters in the descriptions of Aethiopella littoralis Fernandes \& Mendonça, 2002; Brachystomella ceciliae Fernandes \& Mendonça, 2004; Maricaella duna Mendonça \& Fernandes, 1997 and Rapoportella pitomboi Mendonça \& Fernandes, 1995. Among the collected springtails was verified the good taxonomical representation of the Poduromorpha Order (53\%) in the restinga areas.

KEY WORDS. Brachystomellidae; Hypogastruridae; Neanuridae; Restinga; Tullbergiidae.
\end{abstract}

RESUMO. O presente artigo amplia para 34 o número de espécies de Poduromorpha conhecidas no litoral brasileiro através do registro de Paraxenylla piloua Thibaud \& Weiner, 1997; Xenylla welchi Folsom, 1916; Friesea claviseta (Axelson, 1900); Friesea magnicornis Denis, 1931 e Friesea mirabilis (Tullberg, 1871) no litoral de Maricá, estado do Rio de Janeiro. $O$ estudo morfológico das 23 espécies encontradas permitiu anexar caracteres às descrições de Aethiopella littoralis Fernandes \& Mendonça, 2002; Brachystomella ceciliae Fernandes \& Mendonça, 2004; Maricaella duna Mendonça \& Fernandes, 1997 e Rapoportella pitomboi Mendonça \& Fernandes, 1995. Dentre o total de colêmbolos coletados, foi constatada a boa representatividade taxonômica de Poduromorpha (53\%) em áreas de restinga. PALAVRAS-CHAVE. Brachystomellidae; Hypogastruridae; Neanuridae; Restinga; Tullbergiidae.

Estudos envolvendo Poduromorpha no litoral brasileiro eram escassos e relacionados principalmente à família Neanuridae, quando, em 1995 foi criado um Projeto visando ampliar os conhecimentos desta fauna na Restinga de Maricá.

O primeiro registro desta ordem foi feito por ArLé (1959) ao assinalar a presença de Anurida maritima (Guérin, 1838) em faces rochosas do litoral brasileiro. Em um estudo sobre a biologia e ecologia da fauna marinha brasileira realizado por Schuster (1965), foi descrita Pseudanurida sawayana com base em material procedente de mangue localizados em Recife (PE) e em Cananéia (SP) e assinalada a ocorrência de Anurida maritima para a cidade de Recife. Arlé (1966a) descreveu um novo gênero e uma nova espécie, Halachorutes schusteri, abundante na faixa intercotidal entre pedras e cirripédios da região de Salinópolis (PA) e Baía de Sepetiba (RJ). Arlé (1981), em um relato de suas observações realizadas sobre o litoral do Pará, discutiu a bionomia de $H$. schusteri Arlé, 1966, considerando-a talassobionte. Neste mesmo artigo, o autor referiu-se à presença de Pseudanurida sawayana Schuster, 1965 em mangue no Recife (PE), e indicou a ocorrência de Anurida maritima desde Pernambuco até o litoral sul.
O primeiro registro para a Restinga de Maricá foi realizado por Mendonça \& Arlé (1992), ao estudarem a variação quetotáxica de Brachystomella agrosa Wray, 1953, baseada em exemplares coletados na região.

Thibaud \& Palacios-Vargas (1999) ampliaram o conhecimento das espécies ocorrentes no litoral dos estados do Rio de Janeiro e Espírito Santo, através do registro de nove espécies de Poduromorpha: Ceratophysella bengtssoni (Agren, 1904); Mesogastrura cf. ojcoviensis (Stach, 1918); Austrogastrura travassosi (Arlé, 1939); Willemia brevispina Hüther, 1962; Friesea cubensis Potapov \& Banasko, 1985; Friesea josei Palácios-Vargas, 1986; Fissuraphorura cubanica Rusek, 1991; Mesaphorura yosii (Rusek, 1967) e Mesaphorura sp. gr. atlantica.

Importante contribuição para o conhecimento da diversidade e distribuição de Collembola no Brasil foi feita por CuLIK \& Zeppelini-Filho (2003), na qual uma compilação bibliográfica até maio de 2002 apontou a ocorrência de 199 espécies de colêmbolos, sendo a maioria registrada para os estados do Amazonas e Rio de Janeiro. Ainda neste artigo, os autores incluíram uma lista completa das espécies com suas respectivas referências, distribuição e hábitat onde foram encontradas. 
Em 1995 foi criado o Projeto "Estudo Taxonômico e Ecológico de Collembola (Insecta) da Restinga de Maricá/Itaipuaçu, Rio de Janeiro, RJ, Brasil" que resultou, em uma primeira instância, nas descrições de Maricaella duna por Mendonça \& Fernandes (1997) e de Aethiopella littoralis por FernANDEs \& MendonçA (2002). Ainda como resultado deste projeto, FernANDEs \& Mendonça (2004) descreveram quatro novas espécies: Mesaphorura maricaensis, Brachystomella ceciliae, Arlesia intermedia e Micranurida fluminensis e registraram a ocorrência de mais 10 espécies na região: Xenylla maritima Tullberg, 1869; Acherontiella globulata Thibaud \& Massoud, 1979; Onychiurus cf. mariapetrae Thibaud, 1993; Mesaphorura amazonica Oliveira \& Thibaud, 1992; Brachystomella contorta Denis, 1931; Brachystomellides compositus Arlé, 1959; Rapoportella pitomboi Mendonça \& Fernandes, 1995; Friesea reducta Denis, 1931; Pseudachorutes difficilis Denis, 1931; Hylaeanura infima (Arlé, 1959).

\section{MATERIAL E MÉTODOS}

O presente trabalho foi conduzido na Restinga de Maricá, parte da grande "planície litorânea arenosa quaternária" que ocorre ao longo de toda a costa brasileira (Suguio \& TessLer 1984). Esta área está situada no litoral fluminense a leste da cidade do Rio de Janeiro, sob as coordenadas geográficas $22^{\circ} 57^{\prime} 50^{\prime \prime} \mathrm{S}$ e $42^{\circ} 50^{\prime} 44^{\prime \prime}$ W. O clima da região de Maricá é considerado Aw pelo sistema de Köppen, apresentando verão quente e chuvoso e inverno sub-seco. Segundo Nimer (1977) o clima também pode ser considerado do tipo tropical quente superúmido com subseca. Apresenta médias anuais de temperatura máxima de $28^{\circ} \mathrm{C}$ e mínima de $15,3^{\circ} \mathrm{C}$, sendo a temperatura média de $23,6^{\circ} \mathrm{C}$; valores médios mensais de umidade relativa do ar variando de $78,3 \%$ a $84 \%$; total médio anual de precipitação pluviométrica de 1130,8 mm e insolação média de 188,6 horas (MANTOVAn \& Iglesias 2001). O solo da Restinga de Maricá se caracteriza pelo alto conteúdo de areia, baixa percentagem de argila (menos de $0,5 \%)$ e de nutrientes.

No município de Maricá foram escolhidas duas áreas protegidas pela legislação e duas impactadas pela atuação antrópica, sendo cada uma delas situada entre as praias de Itaipuaçu e Barra de Maricá, respectivamente nos distritos de Itaipuaçu e Maricá. As áreas preservadas pela legislação ambiental, estão situadas na Área de Proteção Ambiental (APA) de Maricá, criada através do Decreto Estadual n 7.230 de 23 de abril de 1984 . Esta APA ocupa uma área total de $8,3 \mathrm{Km}^{2}$ entre as coordenadas $22^{\circ} 52^{\prime}$ a $22^{\circ} 54^{\prime} \mathrm{S}$ e $42^{\circ} 48^{\prime}$ a $42^{\circ} 54^{\prime} \mathrm{W}$.

As áreas estudadas foram divididas em dois ambientes, sendo o primeiro compreendido pela vegetação halófila psamófila reptante e o segundo pelo primeiro cordão arenoso, ambos baseados na classificação de Araújo \& Henriques (1984).

O ambiente de vegetação halófila psamófila reptante consiste em uma faixa livre do alcance das marés, mas ainda sujeita à força das ressacas. A vegetação presente neste ambiente é representada por espécies rasteiras, herbáceas, capazes de conviver com a elevada salinidade, a exposição direta aos raios solares e aos ventos. É uma área pobre em nutrientes, onde são típicas as espécies: Alternanthera maritima (Mart.) A. St.-Hil. (Amaranthaceae), Blutaparon portulacoides (A. St.-Hil.) Mears (Amaranthaceae), Sporobolus virginicus (L.) Kunth (Poaceae), Mariscus pedunculatus (R. Br.) T. Koyama (Cyperaceae), Ipomoea pes-caprae (L.) Sweet, Ipomoea imperati (Valhl) Griseg (Convolvulaceae) (SiLva \& Oliveira 1989).

O primeiro cordão arenoso possui formato irregular, no qual a vegetação predominantemente arbustiva e baixa, é em geral abrigada pelas dunas, formando, em alguns trechos, um emaranhado de difícil penetração (SILVA \& Oliveira 1989). Esta área, adaptada aos efeitos da salinidade do solo e mobilidade do substrato, sofre mais intensamente a ação dos ventos (SILVA \& SOMNER 1984). As espécies típicas desta área são: Eugenia uniflora L. (Myrtaceae), Schinus terebenthifolius Raddi (Anacardiaceae), Clusia lanceolata Cambess., Clusia fluminensis Planch. \& Triana (Clusiaceae), Cereus fernambucensis Lem. (Cactaceae), Allagoptera arenaria (Gomes) Kuntze (Arecaceae) e Neoregelia cruenta (Graham) L. B. Sm. (Bromeliaceae) (Silva \& Oliveira 1989).

Foram efetuadas coletas nas duas estações típicas da região, uma seca (inverno) e outra chuvosa (verão), que corresponderam aos meses de janeiro, junho, julho e dezembro de 2003. Em cada uma das áreas e dos ambientes anteriormente determinados foram realizadas quatro amostras de solo/folhiço no período compreendido entre 10:00 h e 15:00 h. As Estações de coleta (Fig. 1) foram assim designadas:

Foram coletadas 128 amostras padronizadas por meio de uma sonda cilíndrica metálica com $4 \mathrm{~cm}$ de profundidade por $10 \mathrm{~cm}$ de diâmetro. As amostras acondicionadas em sacos plásticos etiquetados foram colocadas no laboratório em funis de Berlese-Tullgren para extração da fauna a uma temperatura de $25^{\circ} \mathrm{C}$, durante seis dias. Os espécimens foram fixados em álcool fervente, e a seguir, triados e identificados sob microscópio estereoscópico e microscópio óptico. O estudo taxonômico da fauna foi realizado com o auxílio de chaves e literatura especializada (Salmon 1964, Massoud 1967, Christiansen \& Bellinger 1980, 1992, Bellinger et al. 1996-2007, Weiner 1996, Jordana et al. 1997, Pomorski 1998, Thibaud et al. 2004). Todo o material estudado foi depositado na Coleção de Collembola do Museu Nacional, Universidade Federal do Rio de Janeiro, sob a sigla $\mathrm{CM} / \mathrm{MNRJ}$.

\section{RESULTADOS E DISCUSSÃO}

Foram coletados 8125 colêmbolos, dos quais, 4264 corresponderam à Ordem Poduromorpha, distribuídos em cinco famílias, 16 gêneros e 23 espécies, caracterizadas a seguir:

\section{Neanuridae}

\section{Friesea claviseta (Axelson,1900)}

Material examinado. Brasil, Rio de Janeiro: Maricá, em ambiente de vegetação halófila psamófila reptante de área preservada. L.H. Fernandes \& E.A. Abrantes leg. MAA: $n^{\circ} 1312,1$ ex., 25/VI/2003. 


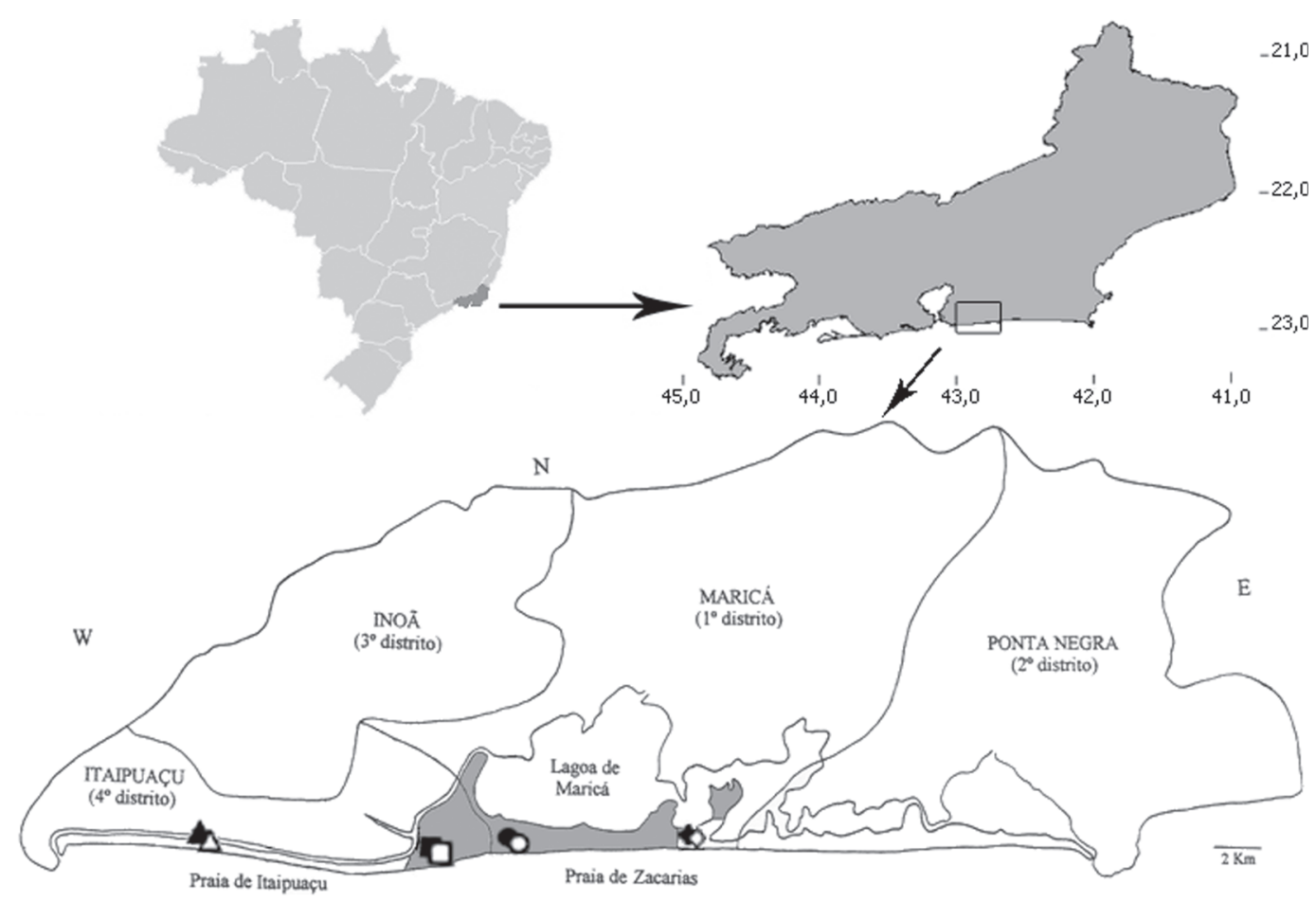

Figura 1. Mapa do município de Maricá (RJ) com delimitação dos Distritos, da APA de Maricá (em cinza) e pontos de coletas: $(\triangle$ ) IPA Praia de Itaipuaçu, vegetação halófila psamófila reptante; $(\mathbf{A})$ IPB - Praia de Itaipuaçu, vegetação de primeiro cordão arenoso; ( $\square$ ) IAA - Itaipuaçu-APA, vegetação halófila psamófila reptante; (ロ) IAB - Itaipuaçu-APA, vegetação de primeiro cordão arenoso; (O) MAA Maricá-APA, vegetação halófila psamófila reptante; $(\bullet)$ MAB - Maricá-APA, vegetação de primeiro cordão arenoso; ( $\diamond)$ MPA - Praia de Barra de Maricá, vegetação halófila psamófila reptante; $($ MPB - Praia de Barra de Maricá, vegetação de primeiro cordão arenoso.

Comentários. Friesea claviseta, espécie hemiedáfica e de distribuição cosmopolita (FJELlBERg 1985, JoRDANA et al. 1997), é registrada pela primeira vez no Brasil. O exemplar coletado na Restinga de Maricá concordou com as descrições e ilustrações de Grow \& Christiansen (1974) e Jordana et al. (1997), porém sem o dente na crista interna da unha referido por THIBAUD \& NAJT (1993) e JoRDANA \& AsiaIn (1981) em material proveniente, respectivamente, do Atol de Fangataufa (Polinésia Francesa) e de Navarra (Espanha).

\section{Friesea magnicornis Denis, 1931}

Material examinado. Brasil, Rio de Janeiro: Maricá, em ambiente de vegetação halófila psamófila reptante de área impactada. L.H. Fernandes \& E.A. Abrantes leg. IPA: $n^{\circ} 1256,1$ ex., 28/I/2003.

Comentários. Friesea magnicornis, espécie hemiedáfica e de distribuição neotropical, é registrada pela primeira vez no Brasil. O material aqui estudado correspondeu às descrições e ilustrações de Denis (1931) e Massoud \& Thibaud (1980), sendo, portanto, uma espécie bem caracterizada morfologicamente.

\section{Friesea mirabilis (Tullberg, 1871)}

Material examinado. Brasil, Rio de Janeiro: Maricá, em ambiente de vegetação halófila psamófila reptante de áreas impactadas e preservadas. L.H. Fernandes \& E.A. Abrantes leg. MAA: $n^{\circ} 1314,1$ ex., 25/VI/2003; MPA: $n^{\circ} 1280$, 3exs; $n^{\circ} 1281$, 18 exs, 28/I/2003; n ${ }^{\circ} 1320,2$ exs, 25/VI/2003; n ${ }^{\circ} 1359,2$ exs, $n^{\circ} 1360,1$ ex., 31/VII/2003.

Comentários. Friesea mirabilis, espécie hemiedáfica e de distribuição provavelmente cosmopolita (JoRDANA et al. 1997), é registrada pela primeira vez no Brasil. Os exemplares coletados concordaram perfeitamente com as descrições e ilustrações de Grow \& Christiansen (1974) e Jordana et al. (1997).

\section{Friesea reducta Denis, 1931}

Material examinado. BrasiL, Rio de Janeiro: Maricá, em ambiente de primeiro cordão arenoso de áreas impactadas e preservadas. L.H. Fernandes \& E.A. Abrantes leg. IPB: $n^{\circ} 1258,2$ exs; $n^{\circ}$ 1259, 38 exs, 28/I/2003; no 1339, 13 exs; no 1342, 5 exs, 31/VII/ 2003; no 1423, 4 exs; $n^{\circ} 1424,2$ exs; $n^{\circ} 1425,5$ exs; $n^{\circ} 1426,6$ exs, 09/XII/2003; IAB: $\mathrm{n}^{\circ}$ 1267, 3 exs; $\mathrm{n}^{\circ}$ 1268, 2 exs, 28/I/2003; $\mathrm{n}^{\circ} 1310$, 3 exs, 25/VI/2003; $n^{\circ} 1431,18$ exs; $n^{\circ} 1432,13$ exs, 09/XII/2003; MAB: $n^{\circ} 1274,4$ exs; $n^{\circ} 1275,16$ exs; $n^{\circ} 1276,12$ exs; $n^{\circ} 1277,2$ exs, 28/I/2003; $n^{\circ} 1356,8$ exs; $n^{\circ} 1358,4$ exs, 31/VII/2003; $n^{\circ} 1440$, 5 exs; $n^{\circ} 1442,1$ ex., 09/XII/2003; MPB: $n^{\circ} 1284,1$ ex.; $n^{\circ} 1285,6$ exs, 28/I/2003; $n^{\circ} 1326,1$ ex., 25/VI/2003; no 1363, 2 exs; $n^{\circ} 1364$, 13 exs, 31/VII/2003; no 1449, 3 exs; $n^{\circ} 1450,3$ exs, 09/XII/2003. 
Comentários. Friesea reducta, espécie hemiedáfica e de distribuição neotropical, já havia sido registrada em substratos de dunas na Restinga de Maricá (Fernandes \& Mendonça 2004). A presença de 4, 5 e 5 ergots, respectivamente, nos tibiotarsos I, II e III, constatada nos exemplares aqui estudados, já havia sido observada por MAssoud \& THIBAUD (1980) em material procedente das Pequenas Antilhas e por Fernandes \& Mendonça (2004) em exemplares da Restinga de Maricá, em oposição à descrição de Denis (1931), onde os mesmos são tidos como ausentes.

\section{Pseudachorutes difficilis Denis, 1931}

Material examinado. BRASIL, Rio de Janeiro: Maricá, em ambiente de primeiro cordão arenoso de áreas impactadas e preservadas. L.H. Fernandes \& E.A. Abrantes leg. IPB: n 1260, 10 exs, 28/I/2003; $\mathrm{n}^{\circ} 1342$, 4 exs, 31/VII/2003; $\mathrm{n}^{\circ} 1424,1$ ex., $\mathrm{n}^{\circ} 1425$, 1 ex., 09/XII/2003; IAB: $n^{\circ} 1269,1$ ex., 28/I/2003; n ${ }^{\circ} 1431,1$ ex.; $n^{\circ} 1432,1$ ex., 09/XII/2003; MAB: $n^{\circ} 1274,1$ ex., $n^{\circ} 1276,3$ exs, 28/I/2003; $n^{\circ} 1440,1$ ex.; $n^{\circ} 1441,1$ ex.; $n^{\circ} 1442,2$ exs, 09/XII/ 2003; MPB: $n^{\circ} 1284,1$ ex.; $n^{\circ} 1285,1$ ex., 28/I/2003; $n^{\circ} 1364,5$ exs; no 1366, 1 ex., 31/VII/2003; n ${ }^{\circ} 1447,1$ ex., 09/XII/2003.

Comentários. Pseudachorutes difficilis, espécie hemiedáfica e de distribuição cosmopolita, já havia sido registrada em substrato de dunas e áreas alagáveis da Restinga de Maricá por Fernandes \& Mendonça (2004). Os exemplares de $P$. difficilis coletados concordaram em todos os aspectos com a revisão do material-tipo realizada por NAjT et al. (1991).

\section{Aethiopella littoralis Fernandes \& Mendonça, 2002}

Material examinado. Brasil, Rio de Janeiro: Maricá, em ambiente de primeiro cordão arenoso de área impactada. L.H. Fernandes \& E.A. Abrantes leg. MPB: $n^{\circ} 1283,1$ ex.; $n^{\circ} 1284,1$ ex.; $n^{\circ} 1285,1$ ex., 28/I/2003; no 1448, 2 exs, 09/XII/2003.

Comentários. Aethiopella littoralis, espécie hemiedáfica, mostrou até o momento uma ocorrência restrita para a Restinga de Maricá. O exame dos exemplares de A. littoralis permitiu ampliar a descrição original de Fernandes \& Mendonça (2002), através dos seguintes caracteres: valvas anais com 15+15 cerdas e $2+2$ cerdas hr e tibiotarsos com cerda $M$ ausente. Também foi constatada a variação de cerdas nos antenômeros I e II, que se apresentaram em número 6-7 e 10-11 cerdas, respectivamente.

\section{Neotropiella sp.}

Material examinado. Brasil, Rio de Janeiro: Maricá, em ambiente de primeiro cordão arenoso de área impactada. L.H. Fernandes \& E.A. Abrantes leg. IPB: no 1424, 1 ex., 09/XII/2003.

Comentários. Devido à presença de um único exemplar de característica hemiedáfica, julgou-se prudente apenas registrar a ocorrência do gênero para a região, uma vez que foi possível visualizar seguramente os seguintes caracteres: coloração geral azulada, com pigmento distribuído uniformemente na cabeça e concentrado em manchas no corpo; quetotaxia do corpo de cerdas finas e lisas; cerdas sensoriais longas e levemente capitadas; fórmula sensorial por meio-tergito: 022/11111; com $5+5$ olhos em placa ocular fortemente pigmentada; órgão pós-antenal moruliforme; maxila estiliforme com lamelas sol- dadas e ápice em crochet; furca normalmente desenvolvida.

\section{Arlesia sp.}

Material examinado. Brasil, Rio de Janeiro: Maricá em ambiente de primeiro cordão arenoso de área impactada, L.H. Fernandes \& E.A. Abrantes leg. MPB: nº1447, 1 ex., 09/XII/2003.

Comentários. Devido à presença de um único exemplar de característica hemiedáfica, julgou-se pertinente apenas registrar sua ocorrência na região de estudo, uma vez que foi possível visualizar seguramente os seguintes caracteres: habitus típico de Pseudachorutinae com paratergitos arredondados; coloração cinza com face ventral, pernas, cone bucal e extremidade das antenas mais claros; quetotaxia do corpo de cerdas finas e lisas, e cerdas sensoriais longas, lisas e levemente capitadas; antenômero IV composto por vesícula apical trilobada, 5 sensilas subcilíndricas; $5+5$ olhos em placa ocular levemente pigmentada; órgão pós-antenal ausente; mandíbula com 4 dentes; maxila estiliforme com lamelas soldadas; furca normalmente desenvolvida; mucro reto com extremidade em crochet.

\section{Hylaeanura infima (Arlé, 1959)}

Material examinado. BRAsIL, Rio de Janeiro: Maricá, em ambiente de primeiro cordão arenoso de área preservada. L.H. Fernandes \& E.A. Abrantes leg. MAB: n 1442, 1 ex., 09/XII/2003.

Comentários. Hylaeanura infima, espécie euedáfica já registrada para a restinga de Itaipuaçu por FeRnandes \& MENDONÇA (2004), teve no presente estudo sua ocorrência estendida para o distrito de Maricá. O único exemplar de H. infima coletado mostrou concordância em todos aspectos com as descrições e ilustrações de Arlé (1959), Arlé (1966b) e Thibaud \& Massoud (1983).

\section{Brachystomellidae}

\section{Brachystomella agrosa Wray, 1953}

Material examinado. BRAsIL, Rio de Janeiro: Maricá, em ambientes de vegetação halófila psamófila reptante e de primeiro cordão arenoso tanto de áreas impactadas quanto preservadas. L.H. Fernandes \& E.A. Abrantes leg. IPA: $n^{\circ} 1254,1$ ex., 28/I/2003; IPB: $n^{\circ} 1260,1$ ex.; $n^{\circ} 1261,43$ exs, 28/I/2003; $\mathrm{n}^{\circ} 1424,1$ ex.; $\mathrm{n}^{\circ} 1425,21$ exs, 09/XII/2003; IAB: $\mathrm{n}^{\circ} 1268,2$ exs; $n^{\circ} 1269,6$ exs, 28/I/2003; MPA: $n^{\circ} 1320,1$ ex., 25/VI/2003; MPB: $n^{\circ} 1282,1$ ex.; $n^{\circ} 1283,1$ ex.; $n^{\circ} 1284,2$ exs; $n^{\circ} 1285,17$ exs, 28/I/2003; no 1364, 13 exs; no 1365, 1 ex., 31/VII/2003.

Comentários. Brachystomella agrosa, espécie hemiedáfica e de distribuição neotropical, já havia sido registrada na área de estudo por Fernandes \& MEndonça (2004). O estudo dos exemplares de B. agrosa procedentes da Restinga de Maricá mostrouse concordante com as descrições e ilustrações de CASSAGNAU \& Rapoport (1962), Massoud \& Thibaud (1980), Najt et al. (1990), Mendonça \& Arlé (1992), Weiner \& Najt (2001). O material aqui examinado confirma as observações anteriormente registradas por Fernandes \& Mendonça (2004) no tocante à presença de numerosas cerdas centrais cefálicas.

\section{Brachystomella ceciliae Fernandes \& Mendonça, 2004}

Material examinado. Brasil, Rio de Janeiro: Maricá, em 
ambientes de vegetação halófila psamófila reptante e de primeiro cordão arenoso tanto de áreas impactadas quanto preservadas. L.H. Fernandes \& E.A. Abrantes leg. IPB: $n^{\circ} 1423,1$ ex., 09/XII/2003; IAB: $n^{\circ} 1267,1$ ex., $n^{\circ}$ 1269, 2 exs, 28/I/2003; $\mathrm{n}^{\circ} 1348,7$ exs, 31/VII/2003; $\mathrm{n}^{\circ} 1431,1$ ex.; $\mathrm{n}^{\circ} 1432,1$ ex., 09/ XII/2003; MAA: $n^{\circ} 1273,2$ exs, 28/I/2003; MAB: $n^{\circ} 1276,5$ exs; $n^{\circ} 1277,1$ ex., 28/I/2003; $n^{\circ} 1356,1$ ex.; $n^{\circ} 1358,1$ ex., 31/VII/2003; $n^{\circ}$ 1440, 1 ex., 09/XII/2003; MPA: no 1280, 1 ex., 28/I/2003; $n^{\circ} 1320,10$ exs, 25/VI/2003; no 1359, 1 ex., 31/VII/ 2003; MPB: $n^{\circ} 1282,1$ ex.; $n^{\circ} 1283,3$ exs, 28/I/2003.

Comentários. A espécie hemiedáfica $B$. ceciliae mostrou até o momento uma ocorrência restrita para a Restinga de Maricá. O exame dos exemplares de B. ceciliae, embora todos ainda jovens, permitiu acrescentar à descrição original de FERNANDEs \& MENDONÇA (2004), os seguintes caracteres: face dorsal da cabeça com as cerdas $\mathrm{a}_{0}, \mathrm{c}_{3}$ e $\mathrm{c}_{5}$ presentes e cerda sd $\mathrm{d}_{1}$ ausente; labro apresentando fórmula 2/2334; valvas anais com $14+14$ cerdas e $3+3$ cerdas hr.

\section{Brachystomella contorta Denis, 1931}

Material examinado. BRASIL, Rio de Janeiro: Maricá, em ambientes de vegetação halófila psamófila reptante e de primeiro cordão arenoso de área impactada. L.H. Fernandes \& E.A. Abrantes col. IPA: $n^{\circ} 1257,13$ exs, 28/I/2003; IPB: $n^{\circ} 1258,14$ exs, 28/I/2003.

Comentários. Brachystomella contorta, espécie hemiedáfica com ampla distribuição geográfica e anteriormente registrada no Brasil por Fernandes \& Mendonça (2004). Os exemplares aqui examinados enquadraram-se perfeitamente nas descrições, revisões e ilustrações de Denis (1931), Christiansen \& Bellinger (1992) e Weiner \& Najt (2001).

\section{Maricaella duna Mendonça \& Fernandes, 1997}

Material examinado. Brasil, Rio de Janeiro: Maricá, em ambiente de primeiro cordão arenoso tanto de área impactada quanto de área preservada. L.H. Fernandes \& E.A. Abrantes leg. IPB: $n^{\circ} 1339,6$ exs; $n^{\circ} 1342,1$ ex., 31/VII/2003; $n^{\circ} 1423,1$ ex., 09/XII/2003; IAB: $n^{\circ} 1268,1$ ex., 28/I/2003; no 1431, 1 ex., 09/ $\mathrm{XII} / 2003$.

Comentários. Maricaella duna, espécie euedáfica, mostrou até o momento uma ocorrência restrita para a Restinga de Maricá. O exame do material de M. duna permitiu ampliar a descrição original de Mendonça \& Fernandes (1997), através dos seguintes caracteres: labro apresentando fórmula 2/2334; cerdas labiais com cerda L em papila, F longa e cerdas E e G menores que as demais; tibiotarso com cerda $M$ presente; apêndice empodial ausente; valvas anais com $13+13$ cerdas e $2+2$ cerdas hr. Também foi constatada a variação no número de cerdas do antenômero II, que se apresentou em número 11-12 cerdas; e no número de cerdas circungenitais da fêmea variando de 4-6.

\section{Rapoportella pitomboi Mendonça \& Fernandes, 1995}

Material examinado. Brasil, Rio de Janeiro: Maricá, em ambientes de vegetação halófila psamófila reptante e de primeiro cordão arenoso tanto de área impactada quanto de área preservada. L.H. Fernandes \& E.A. Abrantes leg. IAA: ${ }^{\circ} 1304,1$ ex., 25/VI/2003; IAB: $n^{\circ} 1432,1$ ex., 09/XII/2003; MPA: ${ }^{\circ} 1279$, 1 ex., 28/I/2003; no 1320, 1 ex., 25/VI/2003.

Comentários. Rapoportella pitomboi, espécie hemiedáfica, foi descrita para o Brasil por MendonçA \& Fernandes (1995) com base em exemplares coletados em Itatiaia (MG) a $2.000 \mathrm{~m}$ de altitude e posteriormente registrada por Fernandes \& MENDOnÇA (2004) na Restinga de Itaipuaçu. O exame dos exemplares de $R$. pitomboi permitiu acrescentar à descrição original de MENDONÇA \& FERnANDES (1995), os seguintes caracteres: cerdas labiais com cerda L em papila; cerda $\mathrm{M}$ ausente no tibiotarso; valvas anais com $11+11$ cerdas e $2+2$ cerdas hr.

\section{Hypogastruridae}

\section{Austrogastrura travassosi (Arlé, 1939)}

Material examinado. BRASIL, Rio de Janeiro: Maricá, em ambiente de vegetação halófila psamófila reptante de áreas impactadas e preservadas. L.H. Fernandes \& E.A. Abrantes leg. MAA: $\mathrm{n}^{\circ} 1354,1$ ex., 31/VII/2003; MPA: $\mathrm{n}^{\circ} 1278,764$ exs; $\mathrm{n}^{\circ}$ 1279, 17 exs; $n^{\circ} 1280,68$ exs; $n^{\circ} 1281,445$ exs, 28/I/2003; $n^{\circ}$ 1319,10 exs; $n^{\circ} 1320,14$ exs, 25/VI/2003; $n^{\circ} 1359,412$ exs, $n^{\circ}$ 1360, 111 exs, 31/VII/2003.

Comentários. Austrogastrura travassosi foi registrada e redescrita por Thibaud \& Palacios-Vargas (1999) com base em material procedente de areias litorâneas de Prainha e Marambaia, Município do Rio de janeiro (RJ). Os exemplares desta espécie euedáfica coletados na restinga concordaram perfeitamente com a revisão desta espécie feita pelos autores acima citados.

\section{Xenylla maritima Tullberg, 1869}

Material examinado. BRASIL, Rio de Janeiro: Maricá, em ambiente de primeiro cordão arenoso de todas as áreas estudadas e no ambiente de vegetação halófila psamófila reptante da área preservada de Itaipuaçu. L.H. Fernandes \& E.A. Abrantes leg. IPB: $\mathrm{n}^{\circ}$ 1259, 1 ex., 28/I/2003; IAA: $n^{\circ} 1265,5$ exs, 28/I/2003; IAB: $n^{\circ}$ 1266, 13 exs; $n^{\circ} 1269,82$ exs, 28/I/2003; $n^{\circ} 1307,1$ ex.; $n^{\circ} 1310$, 9 exs, 25/VI/2003; $n^{\circ}$ 1432, 3 exs, 09/XII/2003; MAB: $n^{\circ} 1274,7$ exs; $n^{\circ} 1276,79$ exs, 28/I/2003; $n^{\circ} 1355,3$ exs; $n^{\circ} 1356,1$ ex.; $n^{\circ}$ 1358, 2 exs, 31/VII/2003; $n^{\circ} 1440,2$ exs; $n^{\circ} 1442,20$ exs, 09/XII/ 2003; MPB: $n^{\circ} 1326,22$ exs, 25/VI/2003; $n^{\circ} 1364,5$ exs; $n^{\circ} 1365$, 56 exs; $n^{\circ} 1366,56$ exs, 31/VII/2003; no 1447, 67 exs; $n^{\circ} 1448,48$ exs; $n^{\circ} 1449,133$ exs; $n^{\circ} 1450,23$ exs, 09/XII/2003.

Comentários. Xenylla maritima, espécie hemiedáfica e de distribuição cosmopolita, foi registrada para o Estado do Rio de Janeiro por Fernandes \& Mendonça (2004). Os exemplares de X. maritima concordaram com as descrições e ilustrações de Tullberg (1869), Axelson (1912), Folsom (1916), Mills (1934), SALMON (1940) e Gama (1964), além das observações feitas por FERNANDES \& MENDONÇA (2004).

\section{Xenylla welchi Folsom, 1916}

Material examinado. Brasil, Rio de Janeiro: Maricá, em ambientes de vegetação halófila psamófila reptante e de primeiro cordão arenoso tanto de área impactada quanto de área 
preservada. L.H. Fernandes \& E.A. Abrantes leg. IAA: $\mathrm{n}^{\circ} 1263,8$ exs; $n^{\circ} 1264,1$ ex., 28/I/2003; n ${ }^{\circ} 1304,15$ exs, 25/VI/2003; IAB: $n^{\circ} 1266,22$ exs; $n^{\circ} 1267,9$ exs; $n^{\circ} 1269,14$ exs, 28/I/2003; $\mathrm{n}^{\circ} 1309,1$ ex.; $\mathrm{n}^{\circ} 1310,1$ ex., 25/VI/2003; $\mathrm{n}^{\circ} 1347,14$ exs; $\mathrm{n}^{\circ}$ 1348, 97 exs, 31/VII/2003; $n^{\circ} 1431,243$ exs; $n^{\circ} 1432,4$ exs; $n^{\circ}$ 1434, 22 exs, 09/XII/2003; MAA: $n^{\circ} 1314,4$ exs, 25/VI/2003; MAB: $n^{\circ} 1276$, 304 exs, 28/I/2003; n ${ }^{\circ} 1356,1$ ex., 31/VII/2003; $n^{\circ} 1442,6$ exs, 09/XII/2003; MPA: $n^{\circ} 1280,3$ exs, 28/I/2003; n 1361, 19 exs, 31/VII/2003.

Comentários. Xenylla welchi, espécie hemiedáfica e de ampla distribuição mundial, havia sido anteriormente registrada para o Brasil no Estado do Espírito Santo por Culik et al. (2002). Os exemplares de $X$. welchi concordaram perfeitamente com a redescrição e ilustrações de Jordana et al. (1997).

\section{Paraxenylla piloua Thibaud \& Weiner, 1997}

Material examinado. Brasil, Rio de Janeiro: Maricá, em ambiente de vegetação halófila psamófila reptante de áreas impactadas e preservadas. L.H. Fernandes \& E.A. Abrantes leg. IAA: $\mathrm{n}^{\circ} 1262,4$ exs; $\mathrm{n}^{\circ} 1263,96$ exs; $\mathrm{n}^{\circ}$ 1264, 6 exs, 28/I/2003; MAA: $n^{\circ} 1272,15$ exs, 28/I/2003; $n^{\circ} 1312,16$ exs; $n^{\circ} 1314,2$ exs, 25/VI/2003; $n^{\circ} 1352,14$ exs; $n^{\circ} 1354,41$ exs, 31/VII/2003; $n^{\circ}$ 1438, 11 exs, 09/XII/2003; MPA: $n^{\circ} 1279,19$ exs; $n^{\circ} 1280,3$ exs; $n^{\circ} 1281,28$ exs, 28/I/2003; no 1319, 2 exs; no 1320, 25 exs, 25/VI/ 2003; no 1360, 78 exs, 31/VII/2003; no 1444, 58 exs, 09/XII/2003.

Comentários. Paraxenylla Murphy, 1965, pela primeira vez encontrado no Brasil, tem distribuição provavelmente mundial nas costas marinhas (JORDANA et al. 1997). A presença de $P$. piloua Thibaud \& Weiner, 1997, espécie hemiedáfica, na área estudada ampliou sua ocorrência, anteriormente só conhecida da Nova Caledônia. Os exemplares de $P$. piloua aqui estudados, corresponderam, de um modo geral, à descrição de Thibaud \& Weiner (1997). No entanto, verificou-se $2+2$ cerdas centrais no tergito torácico II, em vez de 1+1 cerda central, descrita nos exemplares da série-tipo.

\section{Acherontiella globulata Thibaud \& Massoud, 1979}

Material examinado. Brasil, Rio de Janeiro: Maricá, em ambiente de vegetação halófila psamófila reptante de área impactada. L.H. Fernandes \& E.A. Abrantes leg. MPA: ${ }^{\circ} 1279$, 2 exs, 28/I/2003; n 1320, 1 ex., 25/VI/2003.

Comentários. A. globulata, espécie euedáfica descrita por Thibaud \& Massoud (1979) com base material coletado em guano na Ilha de Maria Galante (Pequenas Antilhas), foi registrada no Brasil por Fernandes \& Mendonça (2004). Os três exemplares fêmeas de $A$. globulata estudados, corresponderam em sua totalidade à descrição de Thibaud \& Massoud (1979).

\section{Onychiuridae}

\section{Thalassaphorura sp.}

Material examinado. Brasil, Rio de Janeiro: Maricá, em ambiente de primeiro cordão arenoso de área impactada. L.H. Fernandes \& E.A. Abrantes leg. MPB: n¹448, 1 ex., 09/XII/2003. Comentários. Devido à presença de apenas 1 exemplar coletado, julgou-se prudente apenas registrar a ocorrência deste táxon, de característica euedáfica, na região, uma vez que foi possível observar: habitus alongado e subcilíndrico; coloração branca; quetotaxia formada por microquetas e macroquetas lisas; cerda cefálica $\mathrm{d}_{0}$ presente; sem olhos; órgão pós-antenal simples, com tubérculos dispostos em duas fileiras; peças bucais mastigadoras; antenômero IV com um organito subapical e vesícula apical ausente; órgão sensorial do antenômero III composto por cinco papilas, cinco cerdas de guarda, uma microsensila ventral; tibiotarso com nove cerdas no vértice distal; tenáculo e furca ausentes; área furcal finamente granulada com $2+2$ cerdas arranjadas em duas fileiras; presença de dois espinhos anais.

\section{Tullbergiidae}

\section{Fissuraphorura cubanica Rusek, 1991}

Material examinado. Brasil, Rio de Janeiro: Maricá, em ambiente de vegetação halófila psamófila reptante de área impactada. L.H. Fernandes \& E.A. Abrantes leg. IPA: $n^{\circ} 1255,3$ exs, 28/I/2003; MPA: $\mathrm{n}^{\circ}$ 1278, 24 exs, $\mathrm{n}^{\circ}$ 1279, 6 exs, 28/I/2003.

Comentários. Fissuraphorura cubanica, espécie euedáfica e de distribuição pantropical, já havia sido registrada para o Brasil por Thibaud \& Palacios-Vargas (1999) em areia de praia no Estado do Espírito Santo. Os exemplares de F. cubanica concordaram perfeitamente com a descrição e as ilustrações de RusEK (1991), revelando tratar-se de um táxon bem definido.

\section{Mesaphorura amazonica Oliveira \& Thibaud, 1992}

Material examinado. Brasil, Rio de Janeiro: Maricá, em ambientes de vegetação halófila psamófila reptante e de primeiro cordão arenoso tanto de área impactada quanto de preservada. L.H. Fernandes \& E.A. Abrantes leg. IPB: $n^{\circ} 1258,1$ ex., 28/I/ 2003; IAB: $\mathrm{n}^{\circ} 1431,2$ exs; $\mathrm{n}^{\circ} 1432,1$ ex., 09/XII/2003; MAA: $\mathrm{n}^{\circ}$ 1273, 30 exs, 28/I/2003; MAB: no 1274, 1 ex., 28/I/2003.

Comentários. M. amazonica, espécie euedáfica descrita para o estado do Amazonas por OliveiRa \& Thibaud (1992), foi posteriormente registrada para o Estado do Rio de Janeiro por Fernandes \& Mendonça (2004). Os exemplares de M. amazonica concordaram em grande parte com a descrição original de OLIVeira \& Thibaud (1992) e com as observações verificadas por Fernandes \& Mendonça (2004).

\section{Mesaphorura yosii (Rusek, 1967)}

Material examinado. Brasil, Rio de Janeiro: Maricá, em ambientes de vegetação halófila psamófila reptante e de primeiro cordão arenoso tanto de área impactada quanto de área preservada. L.H. Fernandes \& E.A. Abrantes leg. IPB: $\mathrm{n}^{\circ} 1258,5$ exs; $n^{\circ} 1259,3$ exs; $n^{\circ} 1260,1$ ex.; $n^{\circ} 1261,7$ exs, 28/I/2003; $n^{\circ} 1339$, 2 exs; $n^{\circ} 1340,2$ exs; $n^{\circ} 1342,2$ exs, 31/VII/2003; $n^{\circ} 1423,1$ ex.; $n^{\circ} 1424,1$ ex.; $n^{\circ} 1425,2$ exs; $n^{\circ} 1426,3$ exs, 09/XII/2003; IAA: $n^{\circ} 1262,1$ ex., 28/I/2003; IAB: $n^{\circ} 1266,1$ ex.; $n^{\circ} 1268,2$ exs, 28/ I/2003; $n^{\circ} 1431,1$ ex.; $n^{\circ} 1432$, 1 ex., 09/XII/2003; MAB: $n^{\circ}$ 1275, 2 exs, 28/I/2003; no 1440, 1 ex.; $n^{\circ} 1442,2$ exs, 09/XII/ 2003; MPA: $\mathrm{n}^{\circ}$ 1278, 3 exs, 28/I/2003; MPB: $\mathrm{n}^{\circ}$ 1450, 2 exs, 09/ $\mathrm{XII} / 2003$. 
Comentários. Mesaphorura yosii, espécie euedáfica e de distribuição cosmopolita, já havia sido registrada no Brasil para os estados do Rio de Janeiro (Thibaud \& Palacios-Vargas 1999, Fernandes \& Mendonça 2004) e do Amazonas (Oliveira \& Thibaud 1992). Os exemplares de M. yosii corresponderam em sua grande maioria às descrições de Rusek (1971) e de Christiansen \& Bellinger (1992) e às observações de Najt et al. (1990), Oliveira \& Thibaud (1992) e Fernandes \& Mendonça (2004).

\section{CONSIDERAÇÕES FINAIS}

O estudo morfológico das 23 espécies encontradas permitiu ampliar a descrição de Aethiopella littoraliis, Brachystomella ceciliae, Maricaella duna e Rapoportella pitomboi através da inclusão de novos caracteres.

Dentre as famílias ocorrentes no presente estudo, Neanuridae, com nove espécies, foi a mais representativa e teve no gênero Friesea, com quatro espécies, seu melhor representante. A família menos expressiva, Onychiuridae, teve apenas um representante.

As espécies Paraxenylla piloua, Friesea claviseta, F. mirabilis e F. magnicornis foram registradas pela primeira vez para o Brasil, ampliando de 29 para 34 o número de espécies conhecidas no litoral brasileiro. Além destas, Xenylla welchi e Fissuraphorura cubanica, anteriormente registradas para o estado do Espírito Santo, tiveram sua área de ocorrência estendida para o estado do Rio de Janeiro. A espécie Austrogastrura travassosi de ocorrência já conhecida para o Rio de Janeiro, foi encontrada pela primeira vez na Restinga de Maricá.

Paraxenylla piloua, Acherontiella globulata, Austrogastrura travassosi, Fissuraphorura cubanica e Friesea mirabilis tiveram suas distribuições restritas ao ambiente de vegetação halófila psamófila reptante. Outras como Friesea reducta, Maricaella duna, Pseudachorutes difficilis e Aethiopella littoralis mostraram distribuições restritas ao ambiente de primeiro cordão arenoso.

Quanto à presença da fauna de Poduromorpha em áreas impactadas e preservadas verificou-se que Acherontiella globulata, Austrogastrura travassosi, Fissuraphorura cubanica, Friesea mirabilis, Brachystomella agrosa, Brachystomella contorta e Aethiopella littoralis ocorreram apenas em áreas impactadas, ao passo que Xenylla welchi e Mesaphorura amazonica foram encontradas em áreas preservadas.

Não obstante às condições extremas de vida nos solos arenosos, considerados troficamente pobres (HAY \& LACERDA 1984, Weiner \& Thibaud 1991, Thibaud \& Christian 1995) foi constatada a boa representatividade taxonômica de Poduromorpha (53\%) na Restinga de Maricá, confirmando o que já havia sido observado por FERNANDES \& MENDONÇA (2004) quando registraram 39,3\% de indivíduos desta Ordem na Restinga de Itaipuaçu.

Além disto, constatou-se predominância das formas hemiedáficas e euedáficas, respectivamente com 53\% e 47\%, e de um grande número de formas jovens, coincidindo com as informações de Thibaud \& Christian $(1995,1997)$ sobre a fauna colembológica encontrada, de um modo geral, em solos areno- sos. A presença numerosa de formas jovens, verificada no presente estudo, sugere uma estratégia reprodutiva em que um maior número de ovos e um ciclo de vida mais curto são produzidos sob temperaturas elevadas (Joosse 1969 e GrÉGOIRE-W IBO 1979), como a que ocorre em ecossistema de restinga.

Estudos futuros visando o levantamento de Poduromorpha em outras restingas serão de grande importância para estudos de sistemática, comparação da estrutura e composição, bem como, o conhecimento de espécies indicadoras de áreas impactadas e preservadas.

\section{AGRADECIMENTOS}

Ao CNPq pela concessão de bolsa de estudo; à Fundação Carlos Chagas Filho de Amparo a Pesquisa do Estado do Rio de Janeiro (FAPERJ), pelo auxílio financeiro e à Heloísa A. de L. Carvalho do Departamento de Botânica do Museu Nacional/ UFRJ pelo dedicado e indispensável apoio nos trabalhos de campo e valiosas sugestões.

\section{REFERÊNCIAS BIBLIOGRÁFICAS}

Araújo, D.S.D. de \& R.P.B. Henriques. 1984. Análise florística das restingas do Estado do Rio de Janeiro, p. 159-193. In: L.D. DE Lacerda; R. Cerqueira \& B. Turce (Eds). Restingas: origem, estrutura, processos. Niterói, CEUFF, 475p.

ArLé, R. 1959. Generalidades e importância ecológica da ordem Collembola (Apterygota). Atas da Sociedade de Biologia do Rio de Janeiro 3 (2): 4-7.

ArLé, R. 1966a. Um nouveau Collembole Marin du littoral brésilien. Revista Brasileira de Biologia 26 (4): 367-372.

Arlé, R. 1966b. Collemboles d'Amazonie. I. Poduromorphes nouveaux ou peu connus et notes biologiques sur Neotropiella carli (Denis). Boletim do Museu Paraense Emilio Goeldi, Nova Série Zoologia 60: 1-19.

Arlé, R. 1981. Conspecto das espécies brasileiras de Pseudachorutinae, com descrição de uma espécie nova da Colômbia (Insecta, Collembola). Acta Amazonica 11 (3): 583-593.

Axelson, W.M. 1912. Die Apterygotenfauna Finlands. II. Spezieller Teil. Acta Societatis Scientiarum Fennicae 40 (5): $1-359$.

Bellinger, P.F.; K.A. Christiansen \& F. Janssens. 1996-2007. Checklist of the Collembola of the World. Available in the World Wide Web at: http://www.collembola.org [accessed in 05.I.2007]

Cassagnau, P. \& E.H. Rapoport. 1962. Collemboles d'Amerique du Sud. I. Poduromorphes. Biologie d' Amerique Australe 1: 139-184.

Christiansen, K. \& P.F. Bellinger. 1980. The Collembola of Nort America north of the Rio Grande, a analysis taxonomic. Iowa, Grinnell College, 1322p.

Christiansen, K. \& P.F. Bellinger. 1992. Insects of Hawaii. Collembola. Hawaii, Hawaii University Press, vol.15, VIII+445p.

Culik, M.P. \& D. Zeppelini Filho. 2003. Diversity and distribution

Revista Brasileira de Zoologia 24 (3): 777-785, setembro 2007 
of Collembola (Arthropoda: Hexapoda) of Brazil. Biodiversity and Conservation 12: 1119-1143.

Culik, M.P; J.L de Souza \& J.A. Ventura. 2002. Biodiversity of Collembola in tropical agricultural environments of Espírito Santo, Brazil. Applied Soil Ecology 21: 49-58.

DenIS, J.R. 1931. Contributo alla conoscenza del Microgenton di Costa Rica. II. Collemboles de Costa Rica avec une contribuition au species de l'ordre. Bolletino del Laboratoria di Zoologia generale ed agraria della Facoltà agraria di Portici 25: 69-170.

Fernandes, L.H. \& M.C. Mendonça. 2002. Duas novas espécies de Pseudachorutinae (Collembola, Neanuridae) do Brasil. Boletim do Museu Nacional, Nova Série, Zoologia 496: 1-8.

Fernandes, L.H. \& M.C. Mendonça. 2004. Collembola Poduromorpha do litoral de Maricá, Rio de Janeiro, Brasil. Revista Brasileira de Zoologia 21 (1): 15-25.

FJeLlbERG, A. 1985. Recent advances and future needs in the study of Collembola biology and systematics. Quaestiones Entomologicae 21 (4): 559-570.

Folsom, J.W. 1916. North American Collembolous insects of the subfamilies Achorutinae, Neanurinae, and Podurinae. Proceedings of the United States National Museum 50: 477-525.

Gama, M.M. DA. 1964. Colêmbolos de Portugal Continental. Memórias e Estudos do Museu Zoológico da Universidade de Coimbra 292: 1-252.

Grégorre-Wibo, C. 1979. Cycle phénologique de Folsomia quadrioculata en forêt (Insecta: Collembole). Annalles de la Société Royale Zoologique de Belgique 109: 43-65.

Grow, A.B. \& K. Christiansen. 1974. Chaetotaxy in neartic Friesea (Collembola Neanurinae) with notes on taxonomic use of chaetotaxy. Revue d' Écologie et de Biologie du Sol 11 (3): 377-396.

Hay, J.D. \& L.D. De Lacerda. 1984. Ciclagem de nutrientes no ecossistema de restinga, p. 459-473. In: L.D. DE LACERDA; R. Cerqueira \& B. Turce (Eds). Restingas: origem, estrutura, processos. Niterói, CEUFF, 475p.

Joosse, E.N.G. 1969. Population structure of some surface dwelling Collembola in a coniferous forest soil. Netherlands Journal of Zoology 19 (4): 621-634.

Jordana, R. \& A. Asiain. 1981. Estudio de Friesea en Navarra, con descripción de una nueva forma dentro del grupo, Friesea subterranea. Publicaciones de Biologia de la Universidad de Navarra, Serie Zoologica 6: 47-59.

Jordana, R.; J.I. Arbea; C. Simón \& M.J. Luciáñez. 1997. Collembola, Poduromorpha. In: M.A. Ramos (Ed). Fauna Ibérica, Museo Nacional de Ciencias Naturales. Madrid, CSIC, vol. 8, 807p.

Mantovani, A. \& R.R. Iglesias. 2001. Bromélias terrestres na Restinga de Barra de Maricá, Rio de Janeiro: influência sobre o microclima, o solo e a estocagem de nutrientes em ambientes de borda de moitas. Leandra 16: 17-37.
Massoud, Z. 1967. Monographie des Neanuridae, Collemboles Poduromorphes à pièces buccales modifièes. Biologie de l'Amerique Australe 3: 1-399.

Massoud, Z. \& J.-M. Thibaud. 1980. Les Collemboles des Petites Antilles II. - Neanuridae. Revue d'Écologie et Biologie du Sol 17 (4): 591-605.

Mendonça, C. De \& L.H. Fernandes. 1995. Nova espécie de Rapoportella Ellis \& Bellinger, 1973 do Brasil (Collembola: Neanuridae). Boletim do Museu Nacional, Nova Série, Zoologia 360: 1-6.

Mendonça, C. De \& L.H. Fernandes. 1997. A new genus of Brachystomellinae from Brazil (Collembola: Neanuridae). Boletim do Museu Nacional, Nova Série, Zoologia 379: 1-7.

MendonçA, C. DE \& R. ARlé. 1992. Variação quetotáxica em Brachystomella agrosa Wray, 1953 (Collembola, Neanuridae). Boletim do Museu Nacional, Nova Série, Zoologia 351: 1-9.

Mills, H.B. 1934. A monograph of the Collembola Iowa. Iowa, Collegiate Press, 143p.

Najt, J.; J.-M. Thibaud \& S. Jacquemart. 1991. Les Collemboles (Insecta) de l'Archipel des Galápagos I. Poduromorpha. Entomologie 61: 149-166.

Najt, J.; J.-M. Thibaud \& W. Weiner. 1990. Collemboles (Insecta) Poduromorphes de Guyane française. Bulletin du Museu National d'Histoire Naturelle, $4^{\mathrm{e}}$ sér., section A, 12 (1): 95-121.

Nimer, E. 1977. Clima, p. 51-89. In: IBGE (Ed.). Geografia do Brasil. Região Sudeste. Rio de Janeiro, IBGE.

Oliveira, E. \& J.-M. Thibaud. 1992. Notes sur les collemboles de l'Amazonie, Brésil. 1. Hypogastruridae et Onychiuridae, avec la description de deux espèces nouvelles (Collembola). Opuscula Zoologica Fluminensia 95: 1-8.

POMORSKI, R.J. 1998. Onychiuridae of Poland (Collembola: Onychiuridae). Genus (Suppl.): 1-201.

RuseK, J. 1971. Zur Taxonomie der Tullbergia (Mesaphorura) krausbaueri (Börner) und ihrern Verwandten (Collembola). Acta Entomologica Bohemoslovaca 68: 188-206.

Rusek, J. 1991. New tropical Tullbergiinae (Collembola: Onychiuridae). Acta Entomologica Bohemoslovaca 88: 145-155.

SALmON, J.T. 1940. The Collembolan fauna of New Zealand, including a discussion of its distribution and affinities. Transactions of the Royal Society of New Zealand 70: 282431.

Salmon, J.T. 1964. An Index to the Collembola. Bulletin of the Royal Society of New Zealand 7 (1-3): 1-651.

Schuster, R. 1965. Über die Okologie und Artengliederung der thalassobionten Collembolenfauna Brasiliens. Beiträge zur Neotropischen Fauna 4 (3): 191-208.

Silva, J.G. DA \& A.S. DE Oliveira. 1989. A vegetação de Restinga no Município de Maricá, RJ. Acta Botânica Brasileira 3 (2): 253-272.

Silva, J.G. DA \& G.V. Somner. 1984. A vegetação de Restinga na 
Barra de Maricá, RJ, p.217-224. In: L. D. DE LACERDA; R. CERQUEIRA \& B. TuRCQ (Eds.). Restingas: Origem, Estrutura, Processos. CEUFF, 475p.

Suguio, K. \& M.G. Tessler. 1984. Planícies de cordões litorâneos quaternários do Brasil: origem e nomenclatura, p.15-25. In: L.D. De Lacerda; R. Cerqueira \& B. Turce (Eds). Restingas: Origem, estrutura, processos. Niterói, CEUFF, 475p.

Thibaud, J.-M. \& E. Christian. 1995. Essai de synthèse sur les peuplements de Collemboles interstitiels aériens des sables littoraux et continentaux européens et méditerranéens. Bulletin Entomologique de Pologne 64: 207-216.

Thibaud, J.-M. \& E. Christian. 1997. Biodiversity of interstitial Collembola (Insecta) in sand sediments. European Journal of Soil Biology 33 (3): 123-127.

Thibaud, J.-M.; H.-J. Schulz \& M.M.G. Assalino. 2004. Synopses on Palaeartic Collembola. Vol. 4 Hypogastruridae. Abhandlungen und Berichte des Naturkundemuseums Görlitz 75 (2): 1-287.

Thibaud, J.-M. \& J.G. Palacios-Vargas. 1999. Brazilian Collembola from littoral sand with description of Austrogastrura gen. n. and Isotomodes carioca sp. n. [Hypogastruridae; Isotomidae]. Revue Française d'Entomologie (N.S.) 21 (1): 25-31.

Thibaud, J.-M. \& J. NajT. 1993. Les Collemboles (Insecta) de l'atoll de Fangataufa. Bulletin du Museu. National d'Histoire
Naturelle, $4^{\text {e }}$ sér., section A, 15 (1-4): 95-105.

Thibaud, J.-M. \& W.M. Weiner. 1997. Collemboles interstitiels des sables de Nouvelle-Calédonie. In: J. NAJT \& L. MATile (Eds). Zoologia Neocaledonica, vol. 4. Mémoire du Muséum national d'Histoire naturelle 171: 63-89.

Thibaud, J.-M. \& Z. Massoud. 1979. Les Collemboles des Petites Antilles I. - Hypogastruridae et Onychiuridae. Revue d'Écologie et Biologie du Sol 16 (4): 547-567.

Thibaud, J.-M. \& Z. Massoud. 1983. Les Collemboles des Petites Antilles III. - Neanuridae (Pseudachorutinae). Revue d'Écologie et Biologie du Sol 20 (1): 111-129.

Tullberg, T. 1869. On skandinaviska Podurider af Underfamiljen Lipurinae. Akademische Abhandlung: 1-20.

Weiner, W.M. 1996. Generic revision of Onychiurinae (Collembola: Onychiuridae) with a cladistic analysis. Annales de la Societé Entomologique de France (N.S.) 32 (2): 163-200.

Weiner, W.M. \& J.-M. Thibaud. 1991. Un nouveau genre de Tullbergiinae (Collembola) des sables de la région parisienne. Bulletin du Museu. National d'Histoire Naturelle, $4{ }^{\mathrm{e}}$ sér., section A, 13 (1-2): 113-119.

Weiner, W.M. \& J. NAJT. 2001. Species of Brachystomella (Collembola: Brachystomellidae) from the Neotropical region. European Journal of Entomology 98: 387-413.

Recebido em 15.II.2007; aceito em 15.VIII.2007. 